\title{
Arzneimittelexanthem durch Metamizol mit hämorrhagischer leukozytoplastischer Vaskulitis
}

\author{
A. Beinhauer \\ A. Kleinheinz \\ B. M. Hausen
}

\section{Drug Eruption and Leucocytoplastic Vasculitis Due to Metamizol with Demonstration of Acquired Delayed Type Hypersensitivity}

\section{Zusammenfassung}

Nach postoperativer Gabe verschiedener schmerzstillender, antibiotischer, antihypotensiver und sedierender Medikamente entwickelte eine 68-jährige Patientin innerhalb von 24 Stunden nach der Implantation einer Kniegelenksprothese ein makulopapulöses Arzneimittelexanthem. Am 15. Tag nach der Operation trat auf beiden Fußrücken zusätzlich eine leukozytoklastische Vaskulitis auf. Auf eine 10\%ige Verdünnung der verwendeten Metamizol-Tropfen reagierte die Patientin deutlich. Scratchund Epikutantests mit allen im Zusammenhang mit den Hautveränderungen verabreichten Medikamenten führten sowohl im Scratch- als auch im Epikutantest zu einer starken Spätreaktion auf Metamizol. Zu allergischen Reaktionen vom Spättyp auf Metamizol liegen nur wenige Fallbeschreibungen vor. Eine histologisch gesicherte leukozytoklastische Vaskulitis auf dieses Medikament wurde unseres Wissens nach bisher nicht beschrieben.

\section{Abstract}

A 68-year-old lady developed a maculopapular drug eruption one day after a knee-joint implant of her left leg. Additionally, 15 days later, a leucocytoclastic vasculitis appeared. The diluted drop solution of metamizol (10\%) produced a strong response in the $72 \mathrm{~h}$ reading. Scratch as well as epicutaneous tests with all medicaments used during and after the operation solely showed positive delayed reactions to metamizol. To the best of our knowledge no histologically proven leucocyctoclastic vasculitis after use of metamizol has been described so far.

\section{Einleitung}

Unverträglichkeitsreaktionen auf Arzneimittel treten häufig auf, allergische Reaktionen sind wesentlich seltener. Sie manifestieren sich am häufigsten an der Haut. Metamizol, ein nichtsteroidales Analgetikum, Antipyretikum, Antiphlogistikum und Spasmolytikum, kommt in verschiedenen Darreichungsformen zum Einsatz. Am häufigsten ist es Ursache eines fixen Arzneimittelexanthems [1 -8]. Bei den meisten durch Metamizol hervorgerufenen Reaktionen werden Prick-, Intradermal-, Epikutantests und vor allem die orale Provokation herangezogen, um festzustellen, ob der Patient eine Allergie erworben hat oder ob es sich bei den Hautveränderungen nur um eine Unverträglichkeit gehandelt hat. Im Falle des fixen Arzneimittelexanthems erweist sich eine Testung im Herd als notwendig, da die Applikation des Allergens an der unbefallenen Haut in der Regel negativ bleibt. Eine erneute Konfrontation mit dem Agens könnte lebensbedrohliche Folgen haben, wenn wegen fehlender Tests keine Klarheit über eine möglicherweise bestehende Sensibilisierung besteht.

Ein Beispiel mit schwerwiegenden Nebenwirkungen nach Anwendung von Metamizol wird im Folgenden geschildert. 
Wegen einer Gonarthrose des linken Kniegelenks wurde im Juni 2000 bei einer 68-jährigen ehemaligen Raumpflegerin eine Rotationsendoprothese implantiert. Am folgenden Tag trat ein makulopapulöses Arzneimittelexanthem auf. Zunächst war es nur auf die Beine beschränkt, später breitete es sich auf das gesamte Integument aus. Am vierten Tag entschloss man sich, die postoperativ verabreichten Medikamente Bupivacain, Mepivacain, Metamizol, Temazepam, Cefadrin, Erythromycin und Tramadol abzusetzen. Neben der oralen Gabe von Methylprednisolon (80 mg tgl.) und dem Antihistaminikum Clemastin $(3 \times 1 \mathrm{mg})$ wurde auch die Haut behandelt (Clobetasol-17-propionat). Der jedoch weiterhin progrediente Verlauf erforderte am fünften und sechsten Behandlungstag eine Erhöhung der Kortikoidgabe auf $200 \mathrm{mg}$, die Dosis des Antihistaminikums wurde beibehalten. Erst nach einer Woche konnte mit dem Ausschleichen der Medikamente unter Verringerung des topischen Kortikoids begonnen und das Exanthem schließlich unter Kontrolle gebracht werden.

\section{Befund}

Am vierten Tag nach Beginn der Hautveränderungen zeigten sich unter Aussparung des Kopfes erythematöse, bis $2 \mathrm{~cm}$ große, scharf begrenzte und stark juckende Maculae und Papeln, die teilweise konfluierten.

Nach dem Wechsel von Clobetasol-17-propionat auf Hydrocortison-17-butyrat verstärkten sich die Hautveränderungen am linken Knie erneut. Im Narbenbereich imponierten 2-10 mm große, z. T. konfluierende Maculae und Papeln auf einem $5 \times 10 \mathrm{~cm}$ großen Areal. Im weiteren Verlauf waren auch die Beine und das Gesäß betroffen, während es an den Fußrücken zu 2-5 mm großen hämorrhagischen Veränderungen kam. Eine Nierenbeteiligung konnte nicht nachgewiesen werden. Der am 5. Tag angelegte Urinstatus wies statt dessen auf einen Harnwegsinfekt hin.

Im histologischen Bild einer Probe vom linken Fußrücken zeigte sich im Stratum corneum eine korbgeflechtartige Auflockerung und diskrete Akanthose mit wechselnd plump ausgezogenen Reteleisten, im oberen und mittleren Korium ein perivaskulär akzentuiertes, fokal bandartig konfluierendes, interstitielles Entzündungsinfiltrat, bestehend aus Lymphozyten und Histiozyten mit Einschluss zahlreicher eosinophiler Granulozyten. Die eingeschlossenen Gefäße boten eine deutliche Endothelzellenschwellung sowie kleine fibrinoide Nekrosen der Gefäßwand. In der PAS-Färbung bestätigten sich die fibrinoiden Gefäßwandveränderungen, in der Giemsa-Färbung die sehr ausgedehnten perivaskulären Erythrozytenextravasate. Das histologische Bild entsprach in erster Linie einer bereits abgelaufenen, hämorrhagischen leukozytoklastischen Vaskulitis (Dr. Bernadette Schubert, Am Krankenhaus 5, 21614 Buxtehude).
Ein Jahr nach dem Ereignis wurden alle im zeitlichen Zusammenhang mit dem Arzneimittelexanthem eingesetzten Medikamente allergologisch überprüft. Sowohl im i.c.- als auch im Epikutantest blieben alle zehn hauseigenen Lokalanästhetika, darunter auch Bupivacain und Mepivacain negativ. Der eine Woche später durchgeführte Scratch- und Epikutantest mit Metamizol-Tropfen, der Infusions- und Injektionslösung, Tramadol, Theodrenalin, Erythromycin, Temazepam, Natriumdisulfit, Bupivacain, Mepivacain und Propofol verursachte nur im Fall der Infusionslösung (auf 10\% verdünnt) eine leichte, irritative Reaktion. Scratch- und Epikutantest mit den Metamizol-Tropfen (verdünnt auf $10 \%$ in Wasser) wurden jedoch bereits nach 24 Stunden positiv (Abb.1), in der 72-Stunden-Ablesung zeigte sich eine ++-+++-Reaktion. Die Nachtestung mit gereinigtem Metamizol (10\% in Vaseline) erbrachte nach 72 Stunden eine eindeutige +++-Reaktion (Abb.2), sechs Kontrollen verliefen negativ. Weitere Tests wurden von der Patientin abgelehnt.

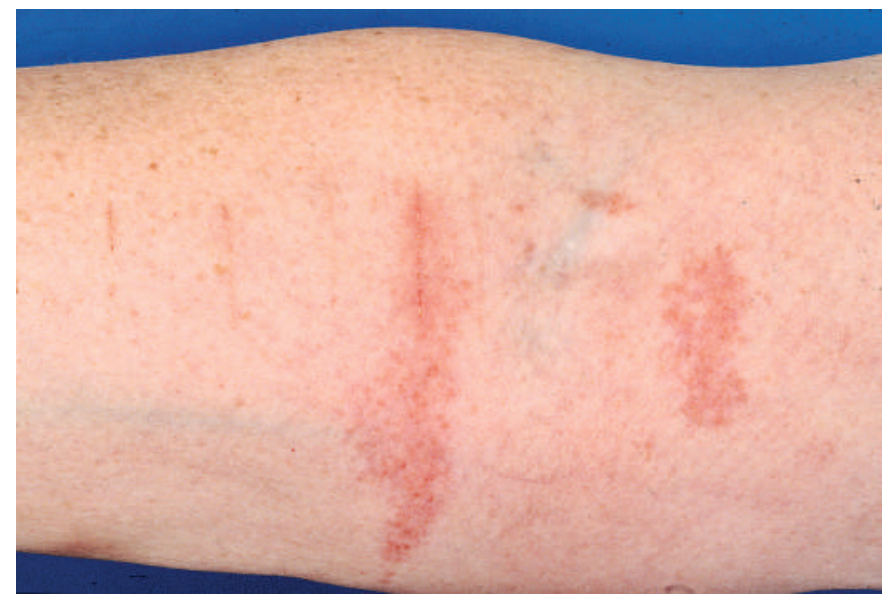

Abb. 1 Spätreaktion auf die Scratchlösung nach 24-Stunden. Durch die Bewegung der Ellenbeuge war die Scratchlösung (Metamizol-Tropfen) auf die gegenüberliegende Armseite übertragen worden, an der sich ebenfalls eine Reaktion entwickelte.

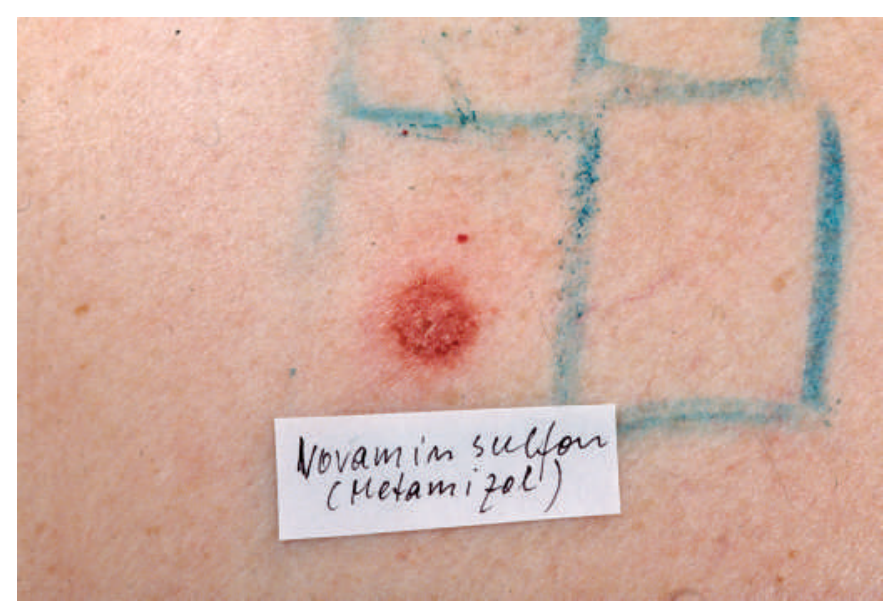

Abb. 2 72-Stunden-Reaktion auf Metamizol (10\% in Vas.) 
Eine echte allergische Spätreaktion auf Metamizol ist relativ selten. Da der Epikutantest (ECT) häufig negativ bleibt, zieht man es heute vor, die Unverträglichkeit durch den Provokationstest zu bestätigen $[8,9]$.

Bernedo et al. [10] berichteten über drei Fälle, bei denen der ECT mit 5\%igem Metamizol positiv wurde. Borja et al. [11] beobachteten drei Patienten mit Hautveränderungen nach oral eingesetztem Metamizol. Zwei zeigten in der 96-Stunden-Ablesung eine positive Testreaktion auf 10\%iges Metamizol. Aus Spanien liegt ein Bericht über ein fixes Arzneimittelexanthem nach oraler und intravenöser Zufuhr bei einem 25-jährigen Mann vor [12]. Ein offener ECT blieb negativ. Fünf Stunden nach dem Okklusivtest an unbefallener Haut und im Herd entwickelte sich jedoch ein generalisierter Juckreiz mit Erythem, nachfolgendem Ödem an den Applikationsstellen und einem Aufflammen der alten Herde an Stamm und Extremitäten. Die Autoren rieten daher zu einem schrittweisen Vorgehen. Sie empfahlen, den Epikutantest mit $1 \%$ zu beginnen und nur bei negativem Ausfall eine höhere Testkonzentration zu verwenden $[8,12]$.

In unserem Fall war Metamizol nicht nur Ursache des anfänglich therapieresistenten Arzneimittelexanthems, sondern höchstwahrscheinlich auch der im weiteren Verlauf aufgetretenen leukozytoklastischen Vaskulitis. In der Nachanamnese berichtete die Patientin über frühere Arzneimittelexantheme, die im Jahre 1988 und 1989 nach Implantation einer Totalendoprothese aufgetreten waren. Nachforschungen ergaben jedoch, dass bei keiner der Operationen Metamizol als Schmerzmittel eingesetzt worden war. Man vermutete vielmehr Aminoglykoside als Ursache der Exantheme, was aufgrund der abgelehnten Testung leider nicht überprüft werden konnte. Auch der behandelnde Hausarzt (seit 1982) und der Orthopäde (seit 1986) verneinten, jemals Metamizol verschrieben zu haben. Die weitere Verfolgung der Spur führte schließlich zu einer Unterleibsoperation im April 1977. Der damals behandelnde Frauenarzt konnte sich noch vage an ein Exanthem erinnern, das wenige Tage nach der Entlassung der Patientin aus dem Krankenhaus aufgetreten war. Die Patientin hatte das bis Mitte der 1980er Jahre frei verkäufliche Metamizol in Tropfenform jederzeit griffbereit auf ihrem Nachttisch stehen. Wahrscheinlich war es auch in diesem Fall wieder einmal von ihr zur Schmerzlinderung eingesetzt worden.
1 Pasricha JS. Drugs causing fixed eruptions. Br J Dermatol 1979; 100 : $183-185$

2 Pandhi RK, Kumar AS, Satish DA et al. Fixed drug eruptions on male genitalia. Sex Transm Dis 1984; 11: 164- 166

${ }^{3}$ Chan HL. Fixed drug eruptions. Int J Dermatol 1984; 23: 607-609

${ }^{4}$ Sharma VK, Dhar S, Gill AN. Drug related involvement of specific sites in fixed drug eruptions. J Dermatol 1996; 23: 530-534

${ }^{5}$ Mahboob A, Haroon TS. Drugs causing fixed eruptions. Int J Dermatol 1998; 37: $833-838$

${ }^{6}$ Zedlitz S, Linzbach L, Kaufmann R et al. Reproducible identification of the causative drug of a fixed drug eruption by oral provocation and lesional patch testing. Contact Dermatitis 2002; 46: $352-353$

7 Özkaya-Bayazit E. Topical provocation in fixed drug eruption due to metamizol and naproxen. Clin Exper Dermatol 2004; 29: 419-422

8 Dalmau J, Serra-Baldrich E, Roé E et al. Use of patch test in fixed drug eruption due to metamizole. Contact Dermatitis 2006; 54: 127-128

${ }^{9}$ Bredlich RO, Gall PRU. Arzneimittelexanthem auf Metamizol-Na. Allergologie 1999; 22: 624-626

${ }^{10}$ Bernedo N, Andicana MT, Uriel O et al. Metamizol as a cause of postoperative erythroderma. Contact Dermatitis 2004; 50: 317-318

${ }^{11}$ Borja JM, Galindo M, Gomez E et al. Delayed skin reactions to metamizol. Allergy 2003; 58: 84-85

${ }^{12}$ Gonzalo-Garijo MA, de Argila D, Rodriguez-Nevado I. Generalized reaction after patch testing with metamizol. Contact Dermatitis 2001; 45: 180

\section{Buchbesprechung}

\section{Häufige Hautkrankheiten im Kindesalter. Klinik, Diagnose, Therapie}

D. Abeck, H. Cremer (Hrsg.)

Darmstadt: Steinkopff, 2006. 147 S. mit zahlreichen Farbfotos. Geb. 64,95€. ISBN 3-7985-1515-8

Die Kinderdermatologie ist ein wichtiges und beliebtes Teilgebiet unseres Faches, das seit langem und vielerorts, auch interdisziplinär, erfolgreich betrieben wird. Wegen der vielen Erbkrankheiten mit Beteiligung der Haut, oft mit ersten diagnoserelevanten Symptomen ebendort, ist die Mitwirkung des klinischen Genetikers von großem Vorteil. Im Alltag stehen aber die häufigen Erkrankungen im Vordergrund, wie dieses Buch zeigt. Das bewährte Herausgeber-Tandem, ein Hautarzt und ein Kinderarzt, stellt die häufigen Hautkrankheiten im Kindesalter, zusammen mit 16 der bewährten Mitautoren vor. Es sind dies entzündliche, allergische und infektiöse Erkrankungen, die in 25 Kapiteln in geordneter Gliederung und guter Bebilderung dargestellt werden. Dazu kommen als Tumoren die kutane Mastozytose und die vaskulären Tumoren mit den Gefäßnävi, sowie ein gesondertes Kapitel über die Therapiebesonderheiten im Kindesalter.

Das Buch dient allen Ärzten, welche Kinder in Praxis und Klinik betreuen, in bewährter Weise. Dabei ist die Beschränkung auf die häufigen Hautkrankheiten im Kindesalter bewusst auf den Gebrauch im praktischen Alltag zugeschnitten und wurde deshalb beibehalten. Das Buch stellt nach wie vor eine gelungene, überarbeitete und erweiterte 3. Fortsetzung der ersten Auflage von 1999 dar, die sich damals gut eingeführt hatte und sicher auch weiterhin einem Bedarf entspricht.

E. G. Jung, Heidelberg 